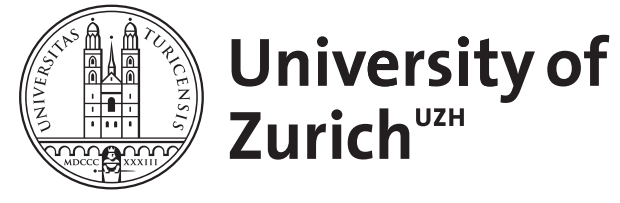

\title{
8 Probiotika und Präbiotika in der Allergieprävention
}

Frei, Remo

DOI: https://doi.org/10.1515/9783110561012-008

Posted at the Zurich Open Repository and Archive, University of Zurich

ZORA URL: https://doi.org/10.5167/uzh-180640

Book Section

Published Version

Originally published at:

Frei, Remo (2019). 8 Probiotika und Präbiotika in der Allergieprävention. In: Schäfer, Torsten; Reese, Imke. Allergieprävention. Berlin: De Gruyter, 97-106.

DOI: https://doi.org/10.1515/9783110561012-008 


\section{Probiotika und Präbiotika in der Allergiepråvention}

Remo Frei

\subsection{Einleitung}

Die Verbreitung von Allergien in Industrieländern hat in den letzten Jahrzehnten stark zugenommen. Mehr als 20\% der Kinder leiden an atopischer Dermatitis [1]. Die atopische Dermatitis ist eine chronische Entzündung der Haut. Tritt sie vor dem 2. Lebensjahr auf, ist das Risiko hoch, dass das Kind im späteren Leben unter anderen atopischen Erkrankungen wie Nahrungsmittelallergien, Asthma und Heuschnupfen leiden wird, was als atopischer Marsch bezeichnet wird [2]. Bis heute ist keine wirksame Prävention gegen die Entstehung von Allergien oder um den atopischen Marsch aufzuhalten bekannt [3]. Neben der Genetik spielen äußere Einflüsse sowohl bei der Entstehung als auch bei der Prävention von Allergien eine wichtige Rolle. Es wird davon ausgegangen, dass die Ernährung von Geburt an eine entscheidende präventive Rolle spielt [4]. Verschiedene Bestandteile der Ernährung interagieren mit der Darmflora und beeinflussen die Metaboliten, die von der Darmflora produziert werden. Die Darmflora und deren Metaboliten sind essentiell für eine gesunde Entwicklung des frühkindlichen Immunsystems [5].

\subsection{Immunologische Grundlagen einer Allergie}

Allergische Symptome werden ausgelöst, wenn ein IgE-Antikörper, der mittels FcERezeptor auf Basophilen oder Mastzellen gebunden ist, ein Allergen mit hoher Affinität bindet [6]. Dadurch wird die Degranulation dieser Zellen induziert, was wiederum Entzündungsmediatoren, z.B. Histamin oder Leukotriene, freisetzt. Diese Entzündungsmediatoren verursachen allergische Symptome wie Kontraktion der Atemwege, erhöhte vaskuläre Durchlässigkeit und Freisetzung zusätzlicher inflammatorischer Zellen [6]. Während der Sensibilisierungsphase werden IgE-Antikörper mit hoher Affinität gegen Allergene gebildet. Epithelzellen bilden als Antwort auf die Exposition gegenüber Allergenen Zytokine wie TSLP (thymic stromal lymphopoietin), Interleukin-25 (IL-25) und IL-33. Diese Zytokine induzieren wiederum eine Typ-2-Immunantwort, die charakterisiert ist durch Rekrutierung von Eosinophilen und Bashophilen, Aktivierung von B-Zellen, dendritischen Zellen, ILC2 (innate lymphoid cells-) und T-Helfer-Zellen Typ $2\left(\mathrm{~T}_{\mathrm{H}} 2\right)$. ILC2 und $\mathrm{T}_{\mathrm{H}}$ 2-Zellen produzieren große Mengen an IL-4, IL-5, IL-9 und IL-13, die den Anitkörperisotypenwechsel zu IgE in B-Zellen induzieren, Eosinophile und Mastzellen rekrutieren, übermäßige Schleimproduktion anregen und Hyperreakivität der Atemwege induzieren [6].

https://doi.org/10.1515/9783110561012-008 


\subsection{Immunologische Toleranz}

Allergische Reaktionen können durch lokale oder systemische Toleranz reduziert oder verhindert werden. Bei der lokalen oder peripheren Toleranz wird eine Immunantwort außerhalb der lymphatischen Organe unterdrückt, ein Prozess, der zum Beispiel bei der Desensibilisierung eine entscheidende Rolle spielt. Toleranz wird durch Anergie von Lymphozyten oder durch Induktion von regulatorischen B- oder T-Zellen vermittelt. Regulatorische T-Zellen exprimieren den Transkriptionsfaktor FOXP3 (forkhead box protein P3) und sezernieren IL-10 und TGF- $\beta$ (transforming growth factor- $\beta$ ) [6].

Neben der genetischen Prädisposition spielen bei der Entwicklung von Allergien und Toleranz auch Umweltfaktoren eine Rolle. Der frühkindliche Kontakt zu Antigenen beispielsweise beeinflusst die Sensibilisierung, aber auch die Entwicklung der Toleranz [6]. Studien mit Migranten zeigen, dass Kinder, die früh im Leben in ein Land mit hoher Allergieprävalenz gezogen sind, ein erhöhtes Risiko haben, an Allergien zu erkranken. Zogen die Kinder später im Leben in ein Land mit hoher Allergieprävalenz, hatte dies keinen Einfluss mehr auf das Allergierisiko [6],[7],[8]. Andere Studien zeigen, dass Kinder, die zwischen dem 4. und 11. Lebensmonat Erdnüsse konsumieren, später weniger an Erdnussallergien leiden [9],[10]. Auf eine Desensibilisierungstherapie für Erdnussallergiker sprachen nur Kinder unter 17 Jahre an; bei den Älteren wurde kein Effekt festgestellt [11].

Die Art des ersten Allergenkontakts spielt ebenfalls eine wichtige Rolle bei der Entscheidung zwischen Toleranz und allergischer Sensibilisierung. Frühkindlicher Allergenkontakt über die Haut ist assoziiert mit Sensibilisierung, während Allergenkontakt über den Gastrointestinaltrakt, vermittelt durch die Ernährung oder durch über die Atemwege aufgenommene Allergene, die in den Darm gelangen, assoziiert ist mit Induktion von Toleranz und regulatorischen T-Zellen [6].

Zusammenfassend kann gesagt werden, dass die Toleranzentwicklung und damit die Induktion von regulatorischen Zellen ein wichtiger immunologischer Mechanismus ist, um das Auftreten von Allergien verhindern. Dabei spielt die frühkindliche Ernährung eine entscheidende Rolle.

\subsection{Darmflora}

Eine gesunde Darmflora hilft Nahrung zu verdauen und aufzunehmen, schützt gegen Kolonisation von Pathogenen, baut Schleim ab und unterstützt die Entwicklung von Epithelzellen und MALTs (mucosal-associated lymphoid tissue) [12]. Es wird aber auch davon ausgegangen, dass eine gesunde Darmflora unabdingbar für eine gesunde Entwicklung der immunologischen Toleranz ist und somit das Fehlen von wichtigen bakteriellen Spezies zu einer inkompletten Entwicklung des Immunsystems führt, was wiederum die Sensibilisierung gegen Allergene verstärkt. Die Kolonisierung des 
Darms mit Mikroben beginnt bei der Geburt, eventuell bereits intrauterin. Die Muttermilch stellt eine große Diversität an Darmbakterien wie Lactobacillus rhamnosus, Lactobacillus gasseri, Lactococcus lactis, Leuconostoc mesenteroides und Bifidobacteria bereit, welche die Entwicklung des Darms beeinflussen. Zusätzlich enthält Muttermilch unverdauliche Oligosaccharide, die das Wachstum von nützlichen Darmbakterien fördern [13]. Andere Faktoren, welche die Darmflora beeinflussen, sind Antibiotikaeinnahme, Hygienische Standards, Geburtsmodus, Ernährungsgewohnheiten und Stadt- versus traditionelles Landleben [6].

Eine veränderte Darmflora führt auch zu einer veränderten metabolischen Aktivität des Darms, was wiederum die Entwicklung der Immuntoleranz beeinflusst [12]. Eine kanadische Studie (Canadian Healthy Infant Longitudinal Development Study) mit 319 Teilnehmer zeigt, dass Kinder, die ein genetisch bedingtes erhöhtes Asthmarisiko haben, in den ersten 100 Lebenstagen eine veränderte Darmflora aufwiesen. Die Bakterientaxa Lachnospira, Veillonella, Faecalibacterium und Rothia waren vermindert, was wiederum zu einer reduzierten Produktion der kurzkettigen Fettsäure Acetat führte [14]. Im Gegensatz dazu fördern Bifidobacterium-, Lactobacillus- und Clostridium-Stämme die Entwicklung von regulatorischen T-Zellen und damit die immunologische Toleranz [12]. Eine weitere Geburtskohortenstudie (Wayne County Health, Environment, Allergy and Asthma Longitudinal Birth Cohort Study) zeigte, dass Kinder, die ein genetisch bedingtes erhöhtes Asthma- und Atopierisiko hatten, weniger Bifidobacterium, Akkermansia und Faecalibacterium, aber mehr der Pilze Candida und Rhodotorula aufwiesen. Zusätzlich wurde eine höhere Konzentration an pro-inflammatorischen Metaboliten gemessen [15]. Eine andere Studie zeigt, dass Kinder mit einer Allergie mehr mit Bacteroides und Klebsiella und weniger mit Clostridium perfringens / butyricum kolonisiert waren [16]. Diese Studien zeigen, dass allergische Erkrankungen mit einem Ungleichgewicht (Dysbiosis) der Darmflora in Zusammenhang stehen.

Zusammenfassend kann gesagt werden, dass die Zusammensetzung und die Diversität der Darmflora mitentscheidend sind für eine gesunde Entwicklung des Immunsystems und der immunologischen Toleranz. Die Zusammensetzung der Darmflora wird durch Umweltfaktoren, aber auch die Genetik und das Mikrobiom der Mutter beeinflusst.

\subsection{Pro- und Präbiotika}

Bei der Geburt ist das Immunsystem naiv. Das heißt, es ist zwar voll ausgebildet, es hatte aber noch keinen Antigenkontakt und hat wenig „Gedächtnis“. Neben der mikrobiellen Kolonisierung unterstützt die Ernährung (Muttermilch oder feste Nahrung) die Entwicklung eines reifen, gesunden Immunsystems, was dazu beiträgt unerwünschte Immunreaktionen, wie sie bei Allergie, Autoimmunerkrankungen oder chronischen Darmentzündungen vorkommen, zu vermindern [13]. 
Der menschliche Verdauungstrakt ist ein hochspezialisiertes System. Das intestinale Immunsystem hat die Aufgabe, die intestinale Integrität $\mathrm{zu}$ bewahren in einem Umfeld, in dem zahlreiche fremde Antigenen vorkommen, die von der Darmflora und der Nahrung präsentiert werden. Ein hochentwickeltes Netzwerk aus Zellen und Molekülen muss dazu fortlaufend koordiniert werden, um einerseits nicht-pathogene Antigene wie Allergene $\mathrm{zu}$ tolerieren, andererseits eine angemessene Immunantwort gegen potenzielle Pathogene bereit zu stellen. Ein aus der Balance geratenes Immunsystem kann chronische Darmentzündungen, Autoimmunerkrankungen und Allergien verursachen [17]. Diese Balance wird durch das Zusammenspiel von angeborenem und adaptivem Immunsystem mit der Darmflora aufrechterhalten. Eine gestörte Kommunikation zwischen Darmflora und Immunsystem aufgrund einer Veränderung der Menge oder Vielfalt der Darmmikroben oder der freigesetzten Metaboliten wird als ursächlich für eine gestörte Balance zwischen Toleranz und Immunantwort angesehen. Die Idee, die Darmflora oder deren Metabolitenproduktion zu modifizieren, hat zur Entwicklung von Probiotika und Präbiotika geführt. Die immunologischen Mechanismen, die durch Pro- und Präbiotika induziert werden, beziehen Epithel-, dendritische- und T-Zellen ein [12],[17].

- Probiotika sind lebendige Mikroorganismen, die, wenn sie in der richtigen Konzentration verabreicht werden, einen vorteilhaften Effekt auf die Gesundheit haben.

- Präbiotika sind fermentierbare Stoffe, welche die Zusammensetzung und die metabolische Aktivität der Darmflora positiv beeinflussen. Typische Präbiotika sind Ballaststoffe, die nicht verdaut, aber von der Darmflora in kurzkettigen Fettsäuren metabolisiert werden können.

- Die Kombination aus Pro- und Präbiotika wird Synbiotika genannt.

- Die freigesetzten Metaboliten der Darmbakterien werden Postbiotika genannt.

\subsubsection{Probiotika}

Probiotika haben einen vorteilhaften Effekt auf die menschliche Gesundheit. Dieser Effekt werden durch 3 Mechanismen vermittelt:

- Probiotika können die Anlagerung oder das Wachstum von Pathogenen verhindern.

- Sie beeinflussen die Integrität der epithelialen Barriere durch Regulation der Schleimproduktion.

- Probiotika beeinflussen die Immunantwort und induzieren Toleranz und haben somit antientzündliche Eigenschaften.

Intestinale dendritische Zellen sind im intestinalen lymphoiden Gewebe, den GALT (gut-associated lymphoid tissues), oder in den intestinalen Lamina propria lokalisiert, wo sie als primäre Immunzellen Kontakt zur Darmflora haben. Dies geschieht 
hauptsächlich durch Rezeptoren des angeborenen Immunsystems (Toll-like Rezeptoren oder C-Typ Lektin Rezeptoren). Dendritische Zellen aktivieren und polarisieren die adaptive Immunantwort durch Zytokin- und Metabolitenproduktion. Bakterielle Signale induzieren eine Phänotyp-Veränderung der dendritischen Zellen und lösen die Sekretion von Zytokinen aus, was die Ansiedelung oder die Abwehr verschiedener Mikroben ermöglicht. Verschiedene klinische Studien zur Behandlung oder Prävention von Allergien mit Probiotika kommen zu widersprüchlichen Resultaten. Neben Faktoren wie dem Studiendesign, den Konzentrationen an Probiotika und verschiedenen Populationen ist die Wahl der Bakterienstämme der essentielle Faktor, der über den Erfolg einer Behandlung entscheidet [16].

Drei Beispiele für Bakterienstämme, die vielversprechende Eigenschaften haben, um als Therapie oder Prävention gegen Allergien zu wirken, sind Lactobacillus rhamnosus GG, Bifidobacterium infantis 35624 und verschiedene Clostridia-Stämme, obwohl auch hier sich widersprechende Studien vorliegen (s. Abb. 8.1). Während Lactobacillus rhamnosus GG einen direkten Effekt auf das Immunsystem hat, brauchen Bifidobacterium infantis 35624 und die Clostridia-Stämme eine geeignete Ernährung als Substrat für die Bildung von Metaboliten, die wiederum die Immunantwort beeinflussen.

Lactobacillus rhamnosus GG (s. Abb. 8.1a)

- Lactobacillus rhamnosus GG ist einer der am meisten verbreiteten Probiotika Stämme. Der Stamm wurde aus Stuhlproben von gesunden Erwachsenen isoliert. Eigenschaften wie Säure- und Galleresistenz, ein gutes Wachstum und Anlagerung an die intestinale Epithelschicht machen ihn $\mathrm{zu}$ einem vielversprechenden Probiotika [18].

- Lactobacillus rhamnosus GG wird mit Prävention und Therapie von gastointestinalen Infektionen und Durchfall in Verbindung gebracht. Zusätzlich reduziert pränatales und postnatales Verabreichen von Lactobacillus rhamnosus GG das Level an totalem IgE und die atopische Sensibilisierung präventiv. Therapeutisch wirkte die Gabe von Lactobacillus rhamnosus $G G$ bei Kindern unter 2 Jahren mit Nahrungsmittelallergien und atopischen Ekzemen und unterstützt die orale Immuntherapie gegen Kuhmilchallergie.

- Durch das Binden von Lipoteichonsäure und unmethylierten Cytosin-Guanin Dinukleotide $(\mathrm{CpG})$ an Toll-like-Rezeptoren wird die Sekretion von Zytokinen wie IL-4, IL-5, IL-10, TGF- $\beta$, TNF- $\alpha$ und IFN-y beeinflusst. Dies verschiebt die Immunantwort von Typ 2 in Richtung Typ 1 [16],[18].

- Lactobacillus rhamnosus GG förderte Autophagie von Epithelzellen und die Schleimproduktion [12]. Autophagie ist eine wichtige Stressantwort, die das Überleben von Zellen sichert und somit entscheidend ist für eine intakte Epithelschicht mit funktionierender Barrierefunktion. Die Schleimschicht im Gastrointestinaltrakt ist ebenfalls wichtig zur Aufrechterhaltung der Barrierefunktion, um unerwünschte Antigene fernzuhalten [17]. 


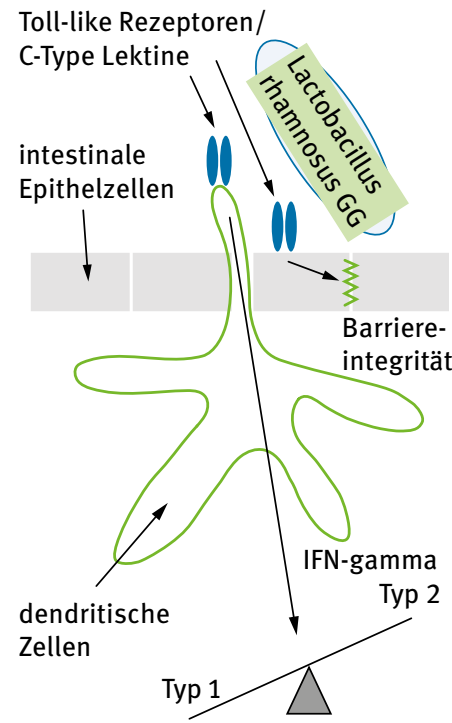

(a)

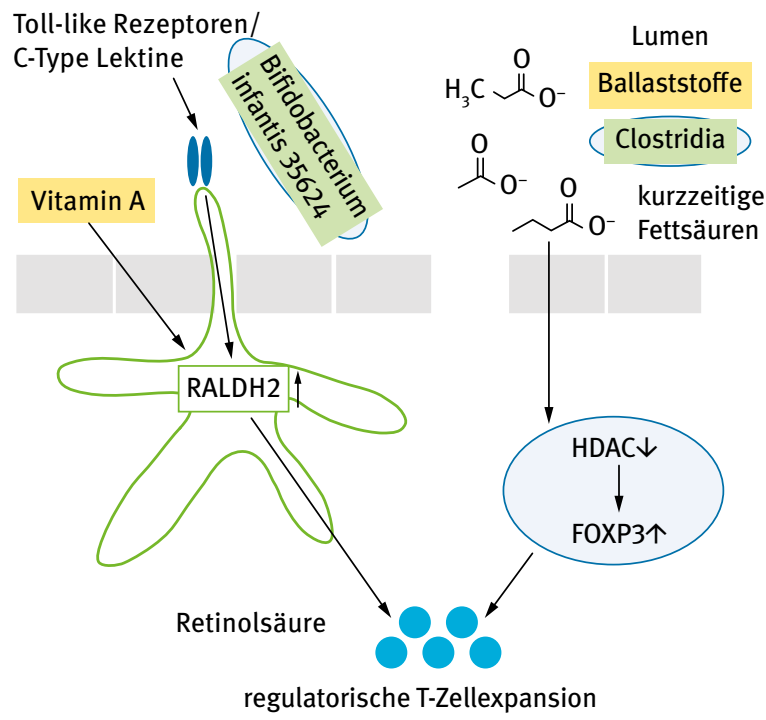

(b)

(c)

Abb. 8.1: Übersicht über die immunologischen Mechanismen, die von Lactobacillus rhamnosus GG (a), Bifidobacterium infantis 35624 (b) und Clostridia (c) induziert werden. HDAC, histone deacetylase.

- Lactobacillus rhamnosus GG förderte das Wachstum anderer Darmbakterien mit immunoregulatorischen Effekten, zum Beispiel Clostrida-Stämmen wie Faecalibacterium, Blautia, Roseburia und Coproccus.

Bifidobacterium infantis 35624 (s. Abb. 8.1b)

- Die Umwandlung von Vitamin A in Retinolsäure ist eine wichtige immunomodulatorische Aktivität, die von intestinalen dendritischen Zellen ausgelöst wird und die Induktion von regulatorischen T Zellen ermöglicht und somit $\mathrm{zu}$ Toleranz führt. Es ist gezeigt worden, dass die Einnahme von Bifidobacterium infantis 35624 RALDH2 (Retinaldehyde dehydrogenase 2) in humanen dendritischen Zellen hochreguliert. RALDH2 wandelt Vitamin A in Retinolsäure um, was regulatorische T-Zellen induziert und so systemische antientzündliche Effekte hat [17],[19],[20]. Die Gabe schützte Mäuse vor der Entwicklung von allergischen Atemwegserkrankungen [12]. Die Induktion von regulatorischen T-Zellen ist ein wesentliches Charakteristikum von protektiven Probiotika [12].

Clostridia-Stämmen (s. Abb. 8.1c)

- Eine Mischung aus 17 Clostridia-Stämmen war in der Lage, die Symptome von Kolitis und allergischem Durchfall zu mindern. Clostridia produzieren in Gegenwart von Ballaststoffen große Mengen an kurzkettigen Fettsäuren, die regulatorische T-Zellen induzieren und somit die immunologische Toleranz fördern (siehe Kapitel 8.5.2) [17]. 
Die Induktion von regulatorischen T-Zellen ist ein Schlüsselmechanismus von protektiven Probiotika. Nicht alle Bakterienstämme sind in dieser Hinsicht gleich effektiv. Eine Studie, bei der verschiedene Stämme (Bifidobacterium longun AH1206, Bifidobacterium breve AH12O5 und Lactobacillus salivarius AH1O2) verglichen wurden, zeigte, dass nur Bifidobacterium longun AH12O6 regulatorische T-Zellen induzierte und somit protektiv gegen die Entstehung von allergischen Atemwegsentzündungen wirkte.

Einen Überblick über 13 randomisierte, placebokontrollierte Studien zeigte, dass gewisse Probiotika-Stämme, aber nicht alle einen präventiven Effekt auf die Allergieentstehung haben [12],[21]. Im Gegensatz dazu zeigte eine Metaanalyse, dass Mikroben für die Behandlung von Allergien weniger effektiv waren [12],[22]. Eine Schwierigkeit könnte darin bestehen, dass der potenteste Stamm für die Induktion der immunologischen Regulation bislang nicht gefunden wurde, da sich nicht alle Stämme in klinischen Studien so verhalten wie in Zellkulturexperimenten. Darüber hinaus ist die metabolische Aktivität der Bakterienstämme wesentlich, um immunologische Toleranz zu induzieren. Die metabolische Aktivität ist aber signifikant abhängig von den bereitgestellten Substraten, also von der Ernährung. Die Ernährung der Probanden wurde in den klinischen Studien mit Probiotika bislang zu wenig berücksichtigt [12].

\subsubsection{Präbiotika}

Ein großer Teil der komplexen Oligosaccharide (Ballaststoffe) aus Früchten, Gemüse oder Cerealien ist unverdaulich für den Menschen, wird aber von Darmbakterien vergärt. Eine ballaststoffreiche Ernährung wird mit verminderten Symptomen von Arthritis, Typ-2-Diabetes, Übergewicht, kolorektalem Karzinom, chronischer Darmentzündung und Allergie assoziiert [4],[12],[23]. Gewisse Ballaststoffe fördern das Wachstum von Bifidobacteria- und Lactobacilli-Stämmen und werden daher als Präbiotika bezeichnet. Präbiotika sind kurzkettige Kohlenhydrate mit einem Polymerisationsgrad zwischen zwei und sechs, beispielsweise Polydextrose, Inulin, Fructo-Oligosaccharide, Glacto-Oligosaccharide und Oligosaccharide von Sojabohnen. Bakterien nutzen den katabolischen Weg (bifid shunt), um aus den Oligosacchariden kurzkettige Fettsäuren und ATP, also Energie, herzustellen. Als erster Schritt wird aus den Oligosacchariden durch Glycosylhydrolasen Fructose-6-phosphat hergestellt, das mittels Phosphoketolase in Phosphoenolpyruvat umgewandelt wird, was wiederum zu Pyruvat degradiert. Pyruvat ist der primäre Metabolit für die kurzkettige Fettsäureproduktion. Pyruvat wird dabei weiter metabolisiert zu Acetyl-CoA, das in Acetat und Butyrat umgewandelt wird. Kurzkettige Fettsäuren werden entweder sezerniert oder in neue Fettsäuren eingebaut, die für zelluläre Strukturen gebraucht werden [12],[24],[25].

Die am häufigsten produzierten kurzkettigen Fettsäuren sind Acetat, Propionat und Butyrat; sie sind wichtig für das Bakterienwachstum im Darm (s. Abb. 8.2). Je 
<smiles>CC(=O)[O-]</smiles>

(a)<smiles>CCC(=O)[O-]</smiles>

(b)<smiles>CCCC(=O)[O-]</smiles>

(c)
Abb. 8.2: Chemische Struktur von Acetat (a), Propionat (b) und Butyrat (c).

nach Oligosacchariden werden verschiedene kurzkettige Fettsäuren produziert. Pektin, Xylan und Galactan werden zu Acetat fermentiert, Arabinoglalactan in Acetat und Propionat umgewandelt und Stärke wird in Butyrat metabolisiert [12]. Kurzkettige Fettsäuren beeinflussen nicht nur die Kolonisation und das Wachstum von Darmbakterien, sondern haben auch einen direkten antientzündlichen Einfluss auf das Immunsystem. Zusätzlich enthalten bestimmt Nahrungsmittel kurzkettige Fettsäuren, zum Beispiel enthalten $100 \mathrm{~g}$ Butter 2,7 g Butyrat und $100 \mathrm{~g}$ Joghurt 0,1 g Butyrat [26],[27].

Butyrat hat stärkere antientzündliche Eigenschaften als Propionat und Acetat [27]. Es fördert die Bildung von regulatorischen T-Zellen, indem es die HDAC (histone deacetylase) inhibiert, was zur erhöhten Acetylierung von Histon H3 im Promotor des Foxp-3-Lokus und damit zur erhöhten Expression des Foxp-3-Gens führt [23],[28],[29],[30]. In dendritischen Zellen wird durch die Inhibition von HDAC die Sekretion der entzündlichen Zytokine IL-12 und IL-6 reduziert und ermöglicht, dass dendritische Zellen die Entstehung von regulatorische T Zellen fördern können. Die G-Protein-gekoppelten Rezeptoren (GPR) 43, GPR41 und GPR109a wurden als Rezeptoren für kurzkettige Fettsäuren beschrieben. Signale von GPR109a in dendritischen Zellen und Makrophagen des Darms resultieren in regulatorischer T-Zell-Induktion und Sekretion des regulatorischen Zytokins IL-10. Signale von GPR43 verbessern die Symptome von Kolitis, Arthritis und allergischen Atemwegserkrankungen [17]. Werden Mäuse mit kurzkettigen Fettsäuren gefüttert, verhindert das weitgehend die Entstehung von allergischen Atemwegserkrankungen und Nahrungsmittelallergien. Eine Beobachtungsstudie an Kindern zeigt, dass diejenigen, die im ersten Lebensjahr hohe Level an Butyrat im Stuhl aufwiesen, im späteren Leben seltener an Allergien litten [27].

Neben kurzkettigen Fettsäuren produzieren Bakterien auch langkettige wie Omega-3-Fettsäuren oder konjugierte Linolsäure. Diese Fettsäuren haben positive Effekte auf Krebserkrankungen, kardiovaskuläre Krankheiten, Diabetes, Übergewicht, Knochen- und Entzündungskrankheiten. Sie besitzen antioxidative Eigenschaften und fördern das Wachstum von Bakterien [12].

Biogene Amine wie Histamin und y-Aminobuttersäure (GABA) werden von Bakterien durch Decarboxylierung von Aminosäuren oder durch Amination oder Transamination von Aldehyden und Ketonen hergestellt [12]. GABA stimuliert die Schleimproduktion in Epithelzellen der Atemwege bei Asthmapatienten. Histamin ist ein wichtiger Mediator von allergischen Symptomen. Die Level an Histamin in den Darmschleimhäuten sind bei Patienten mit chronischer Darmentzündung erhöht und Asthmapatienten weisen mehr Histamin-sezernierende Bakterien im Darm auf [31]. 
Andererseits ist Histamin auch in der Lage, die Sekretion von pro-inflammatorischen Zytokinen durch bakterienstimulierte dendritische Zellen $\mathrm{zu}$ reduzieren und stattdessen die IL-10-Produktion zu induzieren [32].

Das Darmmikrobiom ist wesentlich an der Entwicklung des Immunsystems beteiligt. Gewisse Bakterienstämme sind dabei unabdingbar für die Entwicklung eines gesunden Immunsystems. Andere Stämme sind wichtig für die Entwicklung der immunologischen Toleranz und deshalb besonders geeignet als Probiotika. Die Induktion von regulatorischen T-Zellen scheint ein Schlüsselmechanismus für eine wirksame Allergieprävention und -therapie zu sein. Es wurden bereits probiotische Bakterienstämme identifiziert, die regulatorische T-Zellen induzieren. Dabei scheint der passenden Ernährung eine entscheidende Rolle zuzukommen. Das zeigt das Beispiel von Bifidobacterium infantis 35624: Diese Bifidobakterien produzieren nur Retinolsäure, die für die Induktion regulatorischer T-Zellen verantwortlich ist, wenn auch Vitamin A vorhanden ist. Ein anderes Beispiel sind Clostridia-Stämme, die nur in Gegenwart von ballaststoffreicher Ernährung kurzkettige Fettsäuren bilden können, die dann regulatorische T-Zellen induzieren und damit antientzündliche Eigenschaften haben. Das Ziel der zukünftigen Forschung muss also sein, nicht nur geeignete Bakterien zu identifizieren, sondern auch die dazu passende Ernährung zu finden, um Allergien wirksam verhindern oder therapieren zu können.

\section{Literatur}

[1] Asher MI, Montefort S, Björkstén B, Lai CK, Strachan DP, Weiland SK, et al. Worldwide time trends in the prevalence of symptoms of asthma, allergic rhinoconjunctivitis, and eczema in childhood: ISAAC Phases One and Three repeat multicountry cross-sectional surveys. Lancet. 2006;368(9537):733-743.

[2] Kay J, Gawkrodger DJ, Mortimer MJ, Jaron AG. The prevalence of childhood atopic eczema in a general population. J Am Acad Dermatol. 1994;30:35-39.

[3] Wahn U. Considering 25 years of research on allergy prevention--have we let ourselves down? Pediatr Allergy Immunol. 2013;24(4):308-10.

[4] Thorburn AN, Macia L, Mackay CR. Diet, Metabolites, and "Western-Lifestyle" Inflammatory Diseases. Immunity. 2014;40(6):833-842.

[5] David LA, Maurice CF, Carmody RN, Gootenberg DB, Button JE, Wolfe BE, et al. Diet rapidly and reproducibly alters the human gut microbiome. Nature. 2014;505:559-563.

[6] Reynolds LA, Finlay BB. Early life factors that affect allergy development. Nat. Rev. Immunol.. 2017;17:518-528.

[7] Kuehni CE, Strippoli M-PF, Low N, Silverman M. Asthma in young south Asian women living in the United Kingdom: the importance of early life. Clin Exp Allergy. 2007;

[8] Hjern A, Rasmussen F, Hedlin G. Age at adoption, ethnicity and atopic disorder: A study of internationally adopted young men in Sweden. Pediatr Allergy Immunol. 1999;

[9] Perkin MR, Logan K, Tseng A, Raji B, Ayis S, Peacock J, et al. Randomized Trial of Introduction of Allergenic Foods in Breast-Fed Infants. N Engl J Med. 2016;

[10] Du Toit G, Sayre PH, Roberts G, Sever ML, Lawson K, Bahnson HT, et al. Effect of Avoidance on Peanut Allergy after Early Peanut Consumption. N Engl J Med. 2016;

[11] The PALISADE Group of Clinical Investigators. AR101 Oral Immunotherapy for Peanut Allergy. N Engl J Med. 2018;379(21):1991-2001. 
[12] Frei R, Lauener RP, Crameri R, O'Mahony L. Microbiota and dietary interactions - An update to the hygiene hypothesis? J Allergy Clin Immunol 2012.

[13] Parigi SM, Eldh M, Larssen P, Gabrielsson S, Villablanca E). Breast milk and solid food shaping intestinal immunity. Frontiers in Immunology. 2015.

[14] Arrieta M-C, Stiemsma LT, Dimitriu PA, Thorson L, Russell S, Yurist-Doutsch S, et al. Early infancy microbial and metabolic alterations affect risk of childhood asthma. Sci Transl Med. 2015;

[15] Fujimura KE, Sitarik AR, Havstad S, Lin DL, Levan S, Fadrosh D, et al. Neonatal gut microbiota associates with childhood multisensitized atopy and T cell differentiation. Nat Med. 2016;

[16] Cosenza L, Nocerino R, Di Scala C, Di Costanzo M, Amoroso A, Leone L, et al. Bugs for atopy: The Lactobacillus rhamnosus GG strategy for food allergy prevention and treatment in children. Benef Microbes. 2015.

[17] Frei R, Akdis M, O'mahony L. Prebiotics, probiotics, synbiotics, and the immune system: Experimental data and clinical evidence. Curr Opin Gastroenterol. 2015;

[18] Segers ME, Lebeer S. Towards a better understanding of Lactobacillus rhamnosus GG - host interactions. Microb Cell Fact. 2014;

[19] Groeger D, O’Mahony L, Murphy EF, Bourke JF, Dinan TG, Kiely B, et al. Bifidobacterium infantis 35624 modulates host inflammatory processes beyond the gut. Gut Microbes. 2013;

[20] Konieczna P, Groeger D, Ziegler M, Frei R, Ferstl R, Shanahan F, et al. Bifidobacterium infantis 35624 administration induces Foxp3 T regulatory cells in human peripheral blood: Potenzial role for myeloid and plasmacytoid dendritic cells. Gut. 2012;

[21] Betsi GI, Papadavid E, Falagas ME. Probiotics for the treatment or prevention of atopic dermatitis: A review of the evidence from randomized controlled trials(12.05). Am J Clin Dermatol. 2008;

[22] Boyle RJ, Bath-Hextall F], Leonardi-Bee J, Murrell DF, Tang MLK. Probiotics for treating eczema. Cochrane Database of Systematic Reviews. 2008.

[23] Trompette A, Gollwitzer ES, Yadava K, Sichelstiel AK, Sprenger N, Ngom-Bru C, et al. Gut microbiota metabolism of dietary fiber influences allergic airway disease and hematopoiesis. Nat Med. 2014;

[24] Sela DA, Chapman J, Adeuya A, Kim JH, Chen F, Whitehead TR, et al. The genome sequence of Bifidobacterium longum subsp. infantis reveals adaptations for milk utilization within the infant microbiome. Proc Natl Acad Sci. 2008;

[25] Macfarlane S, Macfarlane GT. Regulation of short-chain fatty acid production. Proc Nutr Soc. 2003;

[26] Parodi PW. Cows' milk fat components as potential anticarcinogenic agents. J Nutr. 1997;

[27] Roduit C, Frei R, Ferstl R, Loeliger S, WestermannP, Rhyner C, et al. High levels of Butyrate and Propionate in early life are associated with protection against atopy. Allergy. 2018.

[28] Smith PM, Howitt MR, Panikov N, Michaud M, Gallini CA, Bohlooly-Y M, et al. The Microbial Metabolites, Short-Chain Fatty Acids, Regulate Colonic Treg Cell Homeostasis. Science 2013;

[29] Furusawa Y, Obata Y, Fukuda S, Endo TA, Nakato G, Takahashi D, et al. Commensal microbederived butyrate induces the differentiation of colonic regulatory T cells. Nature. 2013;

[30] Tan J, McKenzie C, Vuillermin PJ, Goverse G, Vinuesa CG, Mebius RE, et al. Dietary Fiber and Bacterial SCFA Enhance Oral Tolerance and Protect against Food Allergy through Diverse Cellular Pathways. Cell Rep. 2016;

[31] Barcik W, Pugin B, Westermann P, Perez NR, Ferstl R, Wawrzyniak M, et al. Histamine-secreting microbes are increased in the gut of adult asthma patients. J Allergy Clin Immunol. 2016;

[32] Frei R, Ferstl R, Konieczna P, Ziegler M, Simon T, Rugeles TM, et al. Histamine receptor 2 modifies dendritic cell responses to microbial ligands. J Allergy Clin Immunol. 2013; 